\title{
The roles of the cell division cycle-associated gene family in hepatocellular carcinoma
}

\author{
Qiang Tao ${ }^{1,2,3 \#}$, Siliang Chen ${ }^{4 \#}$, Jia Liu ${ }^{5 \#}$, Peng Zhao ${ }^{2,3}$, Lingmin Jiang ${ }^{2,3}$, Xinyue Tu ${ }^{2,3}$, Xiang Tang ${ }^{2,3}$, \\ Zonghao Liu ${ }^{2,3}$, Abudoukeyimu Yasheng ${ }^{1}$, Kahaer Tuerxun ${ }^{1}$, Yun Zheng ${ }^{2,3}$
}

${ }^{1}$ The Second Department of General surgery, The First People's Hospital of Kashi Prefecture, Kashi, China; ${ }^{2}$ State Key Laboratory of Oncology in South China and Collaborative Innovation Center for Cancer Medicine, Sun Yat-Sen University Cancer Center, Guangzhou, China; ${ }^{3}$ Department of Hepatobiliary Oncology, Sun Yat-Sen University Cancer Center, Guangzhou, China; ${ }^{4}$ Department of Hematology, Peking University Shenzhen Hospital, Shenzhen, China; ${ }^{5}$ Department of Neurology, The Seventh Affiliated Hospital of Sun Yat-Sen University, Shenzhen, China

Contributions: (I) Conception and design: Q Tao, S Chen, Y Zheng; (II) Administrative support: A Yasheng, K Tuerxun, Y Zheng; (III) Provision of study materials or patients: A Yasheng, K Tuerxun, Y Zheng; (IV) Collection and assembly of data: Q Tao, S Chen, J Liu; (V) Data analysis and interpretation: Q Tao, S Chen, J Liu; (VI) Manuscript writing: All authors; (VII) Final approval of manuscript: All authors.

\#These authors contributed equally to this work.

Correspondence to: Abudoukeyimu Yasheng. 120 Yingbin Avenue, Kashi 844000, China. Email: 1308111488@qq.com; Kahaer Tuerxun. 120 Yingbin

Avenue, Kashi 844000, China. Email: abdoctor@163.com; Yun Zheng. 651 Dongfeng Road East, Guangzhou 510060, China.

Email: zhengyun@sysucc.org.cn.

Background: The members of the cell division cycle-associated (CDCA) gene family are significant regulators of cell proliferation known to play key roles in various cancers. However, the function of $C D C A$ genes in hepatocellular carcinoma (HCC) is unclear. The aim of this research was to clarify the roles of CDCA family members in HCC using bioinformatics analysis tools.

Methods: We studied data on the mRNA and protein expression of $C D C A$ genes and survival in patients with HCC using the Oncomine, UALCAN, HPA, CCLE, LinkedOmics, cBioPortal, and Metascape databases.

Results: Significant overexpression of all $C D C A$ members was found in HCC tissues. The expression levels of $C D C A$ s were related to the tumor stage, and high expression levels were correlated with a low survival rate in patients with HCC. Also, we observed a high mutation rate (45\%) of CDCAs in the HCC samples, which manifested as deep deletion, amplification, or increased mRNA expression. In the correlation analysis, we found that any $2 C D C A$ members were significantly positively correlated with each other. Cycle-related genes including AHCTF1, AKT1, BIRC5, CENPF, CENPL, and CENPQ were closely associated with CDCA gene alterations.

Conclusions: The findings of this study indicate that CDCAs may be potential therapeutic targets and prognostic indicators for patients with HCC.

Keywords: Hepatocellular carcinoma (HCC); cell division cycle-associated gene family (CDCA gene family); bioinformatics analysis; prognosis

Submitted Jan 26, 2021. Accepted for publication Apr 04, 2021.

doi: 10.21037 /jgo-21-110

View this article at: http://dx.doi.org/10.21037/jgo-21-110 


\section{Introduction}

Hepatocellular carcinoma (HCC) places as the sixth most common cancer and the fourth leading cause of cancerrelated deaths globally (1). In China, the incidence of HCC is $10-20$ per 100,000 , ranking the second of all malignant tumor mortality (2). Although different curative or palliative therapies exist for HCC, the longterm survival rate of patients with HCC is still extremely low (3). The mechanisms of the growth, progression, and metastasis of HCC have been investigated; however, the molecular features of the disease have not yet been identified. Therefore, novel prognostic markers and prospective drug targets need to be discovered to improve the prognosis and individualized treatments for patients with HCC.

The 8 members of the cell division cycle-associated $(C D C A)$ gene family $(C D C A 1-8)$ are significant regulators of cell proliferation. Studies have demonstrated that abnormal expression of $C D C A$ s can cause cancer $(4,5)$. The protein encoded by $C D C A 1$ (also referred to as $N U F 2$ ) is crucial for the nuclear division and stability of microtubules (6). CDCA2 regulates the DNA damage response in the cell cycle by binding to protein phosphatase $1 \gamma(\mathrm{PP} 1 \gamma)(7)$. CDCA3 forms a portion of the ubiquitin ligase (E3) complex SKP1-Cullin-RING-Fbox (SCF), which can degrade the endogenous cell cycle inhibitor WEE1 to regulate the cell cycle (8). CDCA4 is a $\mathrm{G} 1 / \mathrm{S}$ transition-related cell cycle regulator and also modulates p53 expression $(9,10)$. CDCA5 is a key regulator of the cohesion and separation of sister chromatids during cell division (11). CDCA6 (also referred to as CBX2) encodes a polycomb protein complex that maintains the transcriptional repression of multiple genes throughout the growth cycle through chromatin remodeling and histone modification (12). CDCA7 is a cMyc target gene engaged in cMyc-mediated cell transformation (13). Finally, $C D C A 8$ is an essential component of the vertebrate chromosome passenger complex, which has important regulatory involvement in mitosis and cell division (14).

In HCC, the roles of CDCAs are assumed to be complex and distinct. Previous studies of HCC have evidenced the overexpression of $C D C A 3$ and $C D C A 4$, which may participate in cell proliferation, migration, invasion, and apoptosis $(15,16)$. A number of studies have also reported that $C D C A 5$ and $C D C A 8$ play important roles in the development of HCC $(17,18)$. For instance, studies have found $C D C A 5$ to be expressed at high levels in HCC, which has a significant correlation with tumor progression and a poor prognosis $(18,19)$. However, previous studies only focused on several members of $C D C A$ gene family and failed to investigate the expression of this gene family at multiple levels such as tissue and cell. Hence, it is necessary to study the expression of $C D C A$ gene family from multiple levels to understand the individual roles of the whole gene family members including CDCA1-8 in HCC and their potential mechanisms of action.

In the present study, we used online bioinformatics analysis tools to analyze the relationships of $C D C A$ family members with the pathogenesis and progression of HCC, in order to ascertain the expression patterns, underlying functions, and unique prognostic values of $C D C A$ s in HCC. We present the following article in accordance with the MDAR reporting checklist (available at http://dx.doi. org/10.21037/jgo-21-110).

\section{Methods}

\section{Ethics statement}

This research was approved by the institutional ethics committee of Sun Yat-Sen University Cancer Center and was carried out in accordance with the principles of the Helsinki Declaration (as revised in 2013). All data used in this study were retrieved from publically available sources, so there was no requirement to obtain informed participant consent.

\section{Oncomine database analysis}

The Oncomine database (www.oncomine.org), an online cancer microarray database for DNA or RNA sequencing (seq) analysis, was used to investigate expression of $C D C A$ s (20). In the current study, Student's $t$-test was used to compare the transcriptional levels of $C D C A \mathrm{~s}$ in tissues from diverse cancer types and their corresponding normal tissues. The cut-off values for the $\mathrm{P}$ value and fold change were defined as 0.01 and 1.5 , respectively.

\section{UALCAN}

UALCAN (http://ualcan.path.uab.edu/) is an integrative and interactive network resource which can be used to analyze level 3 RNA-seq data and clinical data of 31 different cancers from The Cancer Genome Atlas (TCGA) database. This portal can be used to analyze differences in 
the expression levels of a query gene between tumor and normal samples, and to estimate the influence of a gene's expression level and clinicopathological characteristics on patient survival (21). In our study, we used the portal to evaluate the messenger RNA (mRNA) expression of the $8 C D C A$ family members in HCC tissues as well as their connection with clinicopathological variables of patients with HCC. Differences in transcriptional expression were compared with Student's $t$-test, and $\mathrm{P}<0.01$ was deemed to be statistically significant.

\section{Human Protein Atlas (HPA)}

The HPA (https://www.proteinatlas.org) is an open accessed knowledge resource. All data in it can be retrieved freely to explore the human proteome (22). In this study, we obtained the immunohistochemical (IHC) data of $C D C A$ gene family in HCC and normal tissues for protein level investigation.

\section{Cancer Cell Line Encyclopedia (CCLE)}

CCLE (https://portals.broadinstitute.org/ccle) is a free online database, which compiles chromosomal copy number, large-scale parallel sequencing, and gene expression data of human cancer cell lines (23). In this database, there are about one thousand cell lines data for genomic analysis and visualization. We verified the expression of $C D C A$ gene family in HCC cell lines using datasets downloaded from the CCLE database. Differences in transcriptional expression were compared with Student's $t$-test using GraphPad Prism 9, and $\mathrm{P}<0.01$ was deemed to be statistically significant.

\section{LinkedOmics}

LinkedOmics (http://www.linkedomics.org/login.php) is an open portal website containing multi-omics datasets for all 32 cancer types in TCGA. This portal can be used by biologists and clinicians to access, analyze, and compare multi-omics data within as well as among tumor types (24). In this study, LinkedOmics was used to perform prognostic analyses of the $C D C A$ gene family in patients with HCC.

\section{TCGA database and cBioPortal}

The TCGA database contains sequencing information as well as pathological information on 30 different cancers (25). The cBioPortal for Cancer Genomics (http:// www.cbioportal.org/) is an open source resource that facilitates investigation of multidimensional datasets of cancer genomes (26). The liver HCC (TCGA, Provisional) dataset, which contains information from 371 patients with pathological results, was chosen for further exploration of CDCAs using cBioPortal. The genomic profiles of HCC patients, including the frequency of gene alterations, z-scores of mRNA expression (RNA Seq V2 RSEM), and co-expression and correlations of genes in the CDCA family, were analyzed with the cBioPortal online tool.

\section{Functional enrichment and bioinformatics analysis}

Metascape (http://metascape.org/gp/index.html\#/main/ step1) is an online portal integrating functional enrichment, interactome analysis, gene annotation, and membership search, which utilizes more than 40 bioinformatics knowledgebases (27). In our study, to identify the most frequently altered linked genes, a gene list comprising the $C D C A$ family genes was analyzed with the Kyoto Encyclopedia of Genes and Genomes (KEGG) and Gene Ontology (GO) tools in Metascape.

\section{Statistical analysis}

In the Oncomine database analysis, student's $t$-test was used to compare the transcriptional levels of $C D C A$ s in tissues from diverse cancer types and their corresponding normal tissues. The cut-off values for the $\mathrm{P}$ value and fold change were defined as 0.01 and 1.5 , respectively. In the UALCAN database analysis, differences in transcriptional expression were compared with Student's $t$-test, and $\mathrm{P}<0.01$ was deemed to be statistically significant. In the CCLE database analysis, differences in transcriptional expression were compared with student's $t$-test, and $\mathrm{P}<0.01$ was deemed to be statistically significant. In this study, CCLE database analysis was performed using GraphPad Prism 9, and all other databases analyses were performed with database online tools.

\section{Results}

\section{Transcriptional levels of CDCA genes in patients with HCC}

First, we identified $C D C A$ genes in the human genome. To investigate the different prognostic and potential 
Table 1 The significant changes of CDCA expression in transcription level between different types of HCC and normal samples

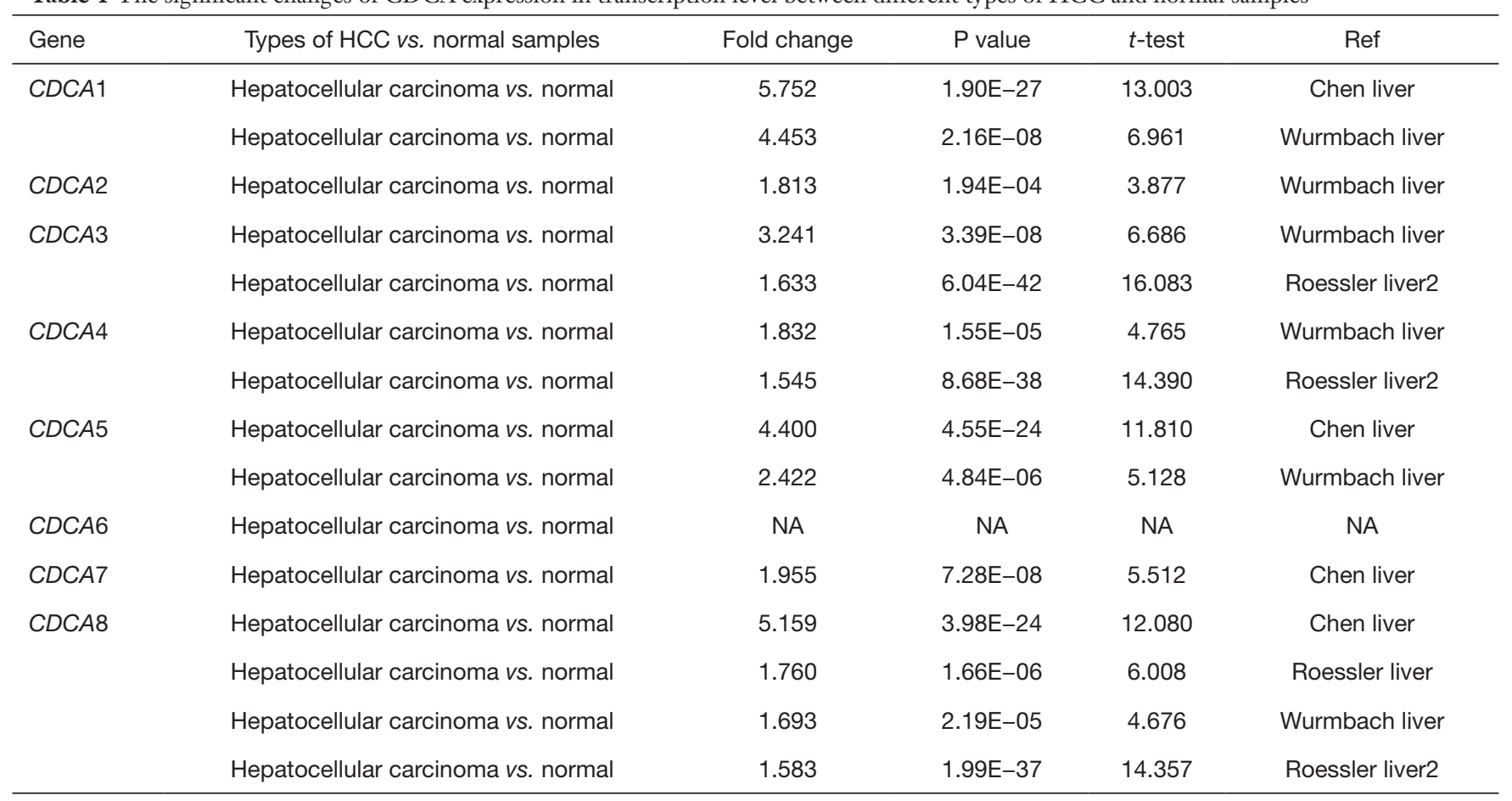

CDCA, cell division cycle-associated; HCC, hepatocellular carcinoma; NA, not available.

therapeutic values of $C D C A$ family members in HCC, the Oncomine database was used to compare the transcriptional levels of the $8 C D C A$ genes between tissue samples from 20 different cancers and samples from normal controls (Table 1 and Figure 1). The mRNA expression levels of $C D C A 1$, $C D C A 2, C D C A 3, C D C A 4, C D C A 5, C D C A 7$, and CDCA8 were upregulated in patients with HCC. In the Chen liver dataset, CDCA1 mRNA was overexpressed 5.752-fold in HCC tissues compared to normal tissues ( $\mathrm{P}=1.90 \mathrm{E}-27)$ (28), while in the Wurmbach liver dataset, it was upregulated in HCC, with a fold change of 4.453 compared to normal tissues $(\mathrm{P}=2.16 \mathrm{E}-08)(29)$. The results of analysis of the Wurmbach liver dataset also showed that the mRNA expression of CDCA2 showed a fold increase of 1.813 in HCC compared to normal tissues $(\mathrm{P}=1.94 \mathrm{E}-04)$ (29). $C D C A 3$ overexpression was also found in HCC: the fold change in the Wurmbach liver dataset was 3.241 $(\mathrm{P}=3.39 \mathrm{E}-08)$, while that in the Roessler liver 2 dataset was $1.633(\mathrm{P}=6.04 \mathrm{E}-42)(29,30)$. The transcriptional expression of $C D C A 4, C D C A 5, C D C A 7$, and $C D C A 8$ was also upregulated in patients with HCC. $C D C A 4$ was identified to be expressed at a higher level in HCC tissues compared to normal tissues in the Wurmbach liver and Roessler liver 2 datasets, with fold changes of 1.832 and
1.545 , respectively $(\mathrm{P}=1.55 \mathrm{E}-05$ and $8.68 \mathrm{E}-38$, respectively) $(29,30)$. Furthermore, $C D C A 5$ was significantly upregulated in HCC, with fold changes of 4.400 and 2.422 in the Chen liver dataset and Wurmbach liver dataset, respectively $(\mathrm{P}=4.55 \mathrm{E}-24$ and 4.84E-06, respectively) $(28,29) . C D C A 7$ was also discovered to have a higher expression in HCC tissues than normal tissues in the Chen liver dataset, with a fold change of 1.955 ( $\mathrm{P}=7.28 \mathrm{E}-08)$ (28). Additionally, overexpression of $C D C A 8$ was also found in HCC, with a fold change of 5.159 in the Chen liver dataset $(\mathrm{P}=3.98 \mathrm{E}-24)$ (28). Meanwhile, Roessler reported 1.760-fold and 1.583fold increases in CDCA8 mRNA expression in HCC $(\mathrm{P}=1.66 \mathrm{E}-06$ and 1.99E-37, respectively), while Wurmbach described a 1.693 -fold rise in CDCA8 mRNA expression in HCC tissues $(\mathrm{P}=2.19 \mathrm{E}-05)(29,30)$. In line with the Oncomine analyses, there was no significant difference in the expression levels of mRNA of CDCA6 between HCC and normal tissues.

Next, the UALCAN database was used to explore differences in the mRNA expression levels of $C D C A \mathrm{~s}$ between HCC and normal tissues. As shown in Figure 2, we found that the expression levels of all $C D C A$ family members in HCC were obviously higher than those in normal tissues (all $\mathrm{P}<0.05)$. 

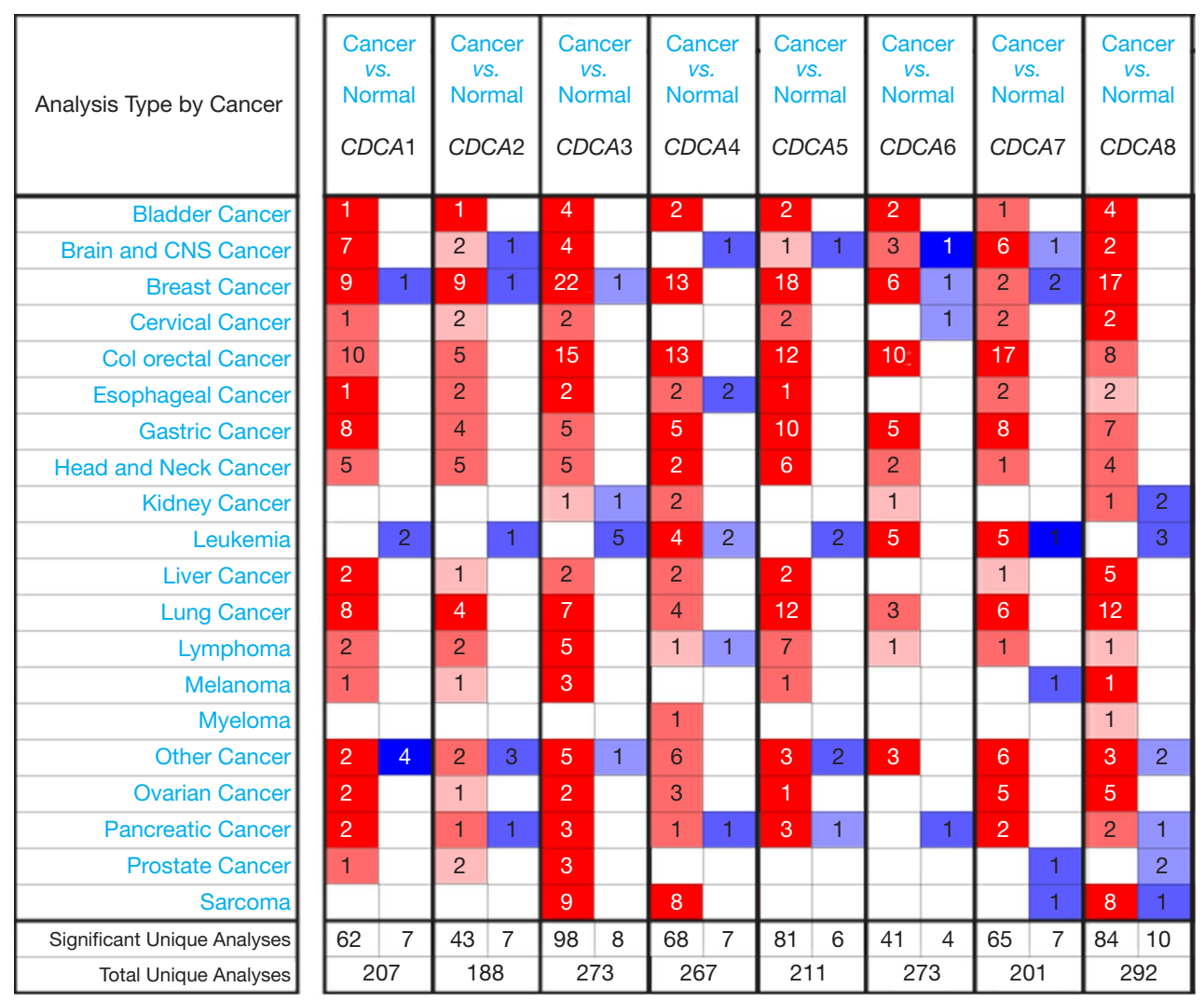

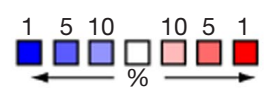

Figure 1 The transcriptional levels of $C D C A$ family members in various cancers. $C D C A$, cell division cycle-associated.

To further verify the prognostic values of $C D C A$ family members in HCC, we used the IHC data from HPA database to compare the protein expression of $C D C A$ genes between HCC tissues and normal tissues. The data of CDCA2, CDCA5, CDCA6 and CDCA8 were available, which showed that these four proteins expressed more highly in HCC tissues than normal tissues (Figure 3).

We also verified the mRNA expression of $C D C A$ genes from cell level with the CCLE database. The results were presented in Figure 4. We found that all CDCA gene family members were highly expressed in the HCC cell lines from CCLE database. We further compared the mRNA expression level of CDCA genes in HCC cell lines before and after knockdown. The mRNA expression data of all $C D C A$ genes knockdown except $C D C A 3$ were available in the CCLE database. The results showed that, in HCC cell lines, $C D C A$ genes knockdown resulted in lower mRNA expression.

\section{Relationship between the clinicopathological variables and CDCA mRNA expression of HCC patients}

After discovering that the mRNAs of all CDCA genes were overexpressed in patients with HCC, we explored the relationships between $C D C A$ s and HCC stage through UALCAN. The mRNA expression levels of $C D C A$ genes were shown to be significantly positively correlated with the stage of HCC (Figure 5). The level of CDCA6 mRNA expression was highest in patients with stage 4 HCC, while the highest expression of other $C D C A$ genes was observed in patients with stage $3 \mathrm{HCC}$.

\section{The role of CDCAs in the survival of HCC patients}

We also analyzed the prognostic values of $C D C A$ s in HCC using LinkedOmics. As shown in Figure 6, the results revealed that elevated expression levels of $C D C A 1-8 \mathrm{mRNA}$ were significantly correlated with short overall survival 

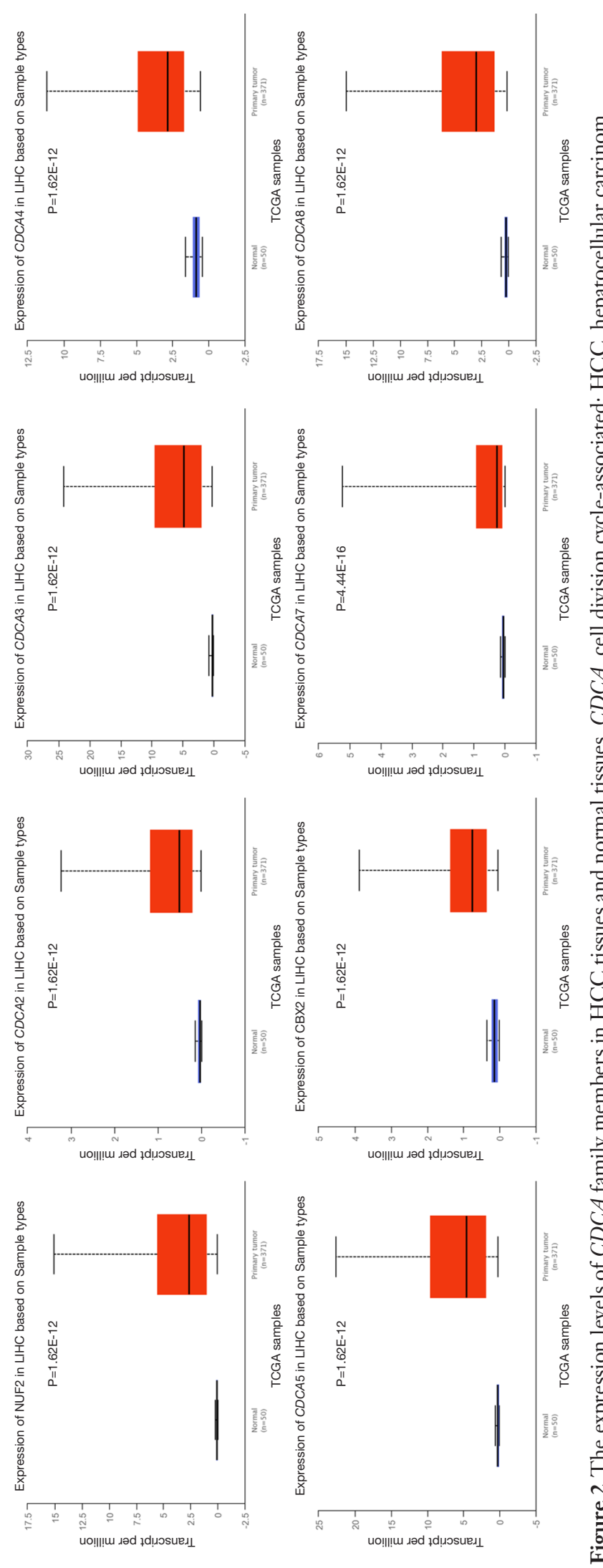

(OS) $(\mathrm{P}<0.05)$ in HCC patients. This result indicated that overexpression of $C D C A 1-8$ may constitute a poor prognostic factor for HCC, and these genes may be used as biomarkers to predict the survival of patients with HCC.

\section{Genetic mutations and correlations of CDCA genes in HCC}

We next analyzed the mutations, correlations, and networks of $C D C A$ genes in HCC using the "TCGA Provisional" database as well as the cBioPortal online tool for HCC. $C D C A$ genes were altered in 167 of $373(45 \%)$ HCC samples. CDCA1, CDCA6, and CDCA2 were the 3 genes that showed the highest genetic variation, with mutation rates of $27 \%, 15 \%$, and $12 \%$, respectively. There were 3 main types of genetic alterations: deep deletion, mRNA overexpression, and amplification (Figure $7 A$ ). We further analyzed the mRNA expression of $C D C A$ genes to examine their correlations with each other using cBioPortal [mRNA sequencing (RNA-seq) version V2 RSEM], together with Pearson's correlation coefficient. We found that any $2 C D C A$ family members were significantly positively correlated with each other (Figure $7 B$ ). Then, we established the gene relation network to show $C D C A$ genes and their the 50 most frequently altered adjacent genes. We found that cell cycle-related genes, such as AHCTF1, $A K T 1, B I R C 5, C E N P F, C E N P L$, and CENPQ, were closely associated with $C D C A$ gene alterations (Figure $7 C$ ).

\section{Predicted functions and pathway enrichment of CDCA genes in $\mathrm{HCC}$}

Before using the GO tools in Metascape, we compiled a list of $C D C A$ genes and 50 neighboring genes that exhibited alterations most frequently (Figure 8 ). The results of enrichment analyses revealed that $C D C A$ gene alterations influenced the following pathways: R-HSA-68886: $M$ Phase; R-HSA-69620: Cell Cycle Checkpoints; GO:1903827: regulation of cellular protein localization; GO:1902850: microtubule cytoskeleton organization involved in mitosis; M139: PID MYC PATHWAY; M14: PID AURORA B PATHWAY; R-HSA-2468052: Establishment of Sister Chromatid Cohesion; R-HSA-5689901: Metalloprotease DUBs; GO:0006997: nucleus organization; R-HSA-8866654: E3 ubiquitin ligases ubiquitinate target proteins; CORUM:1464: Mis 12 centromere complex; R-HSA-432142: Platelet sensitization by LDL; R-HSA-3108232: SUMO E3 ligases SUMOylate target 


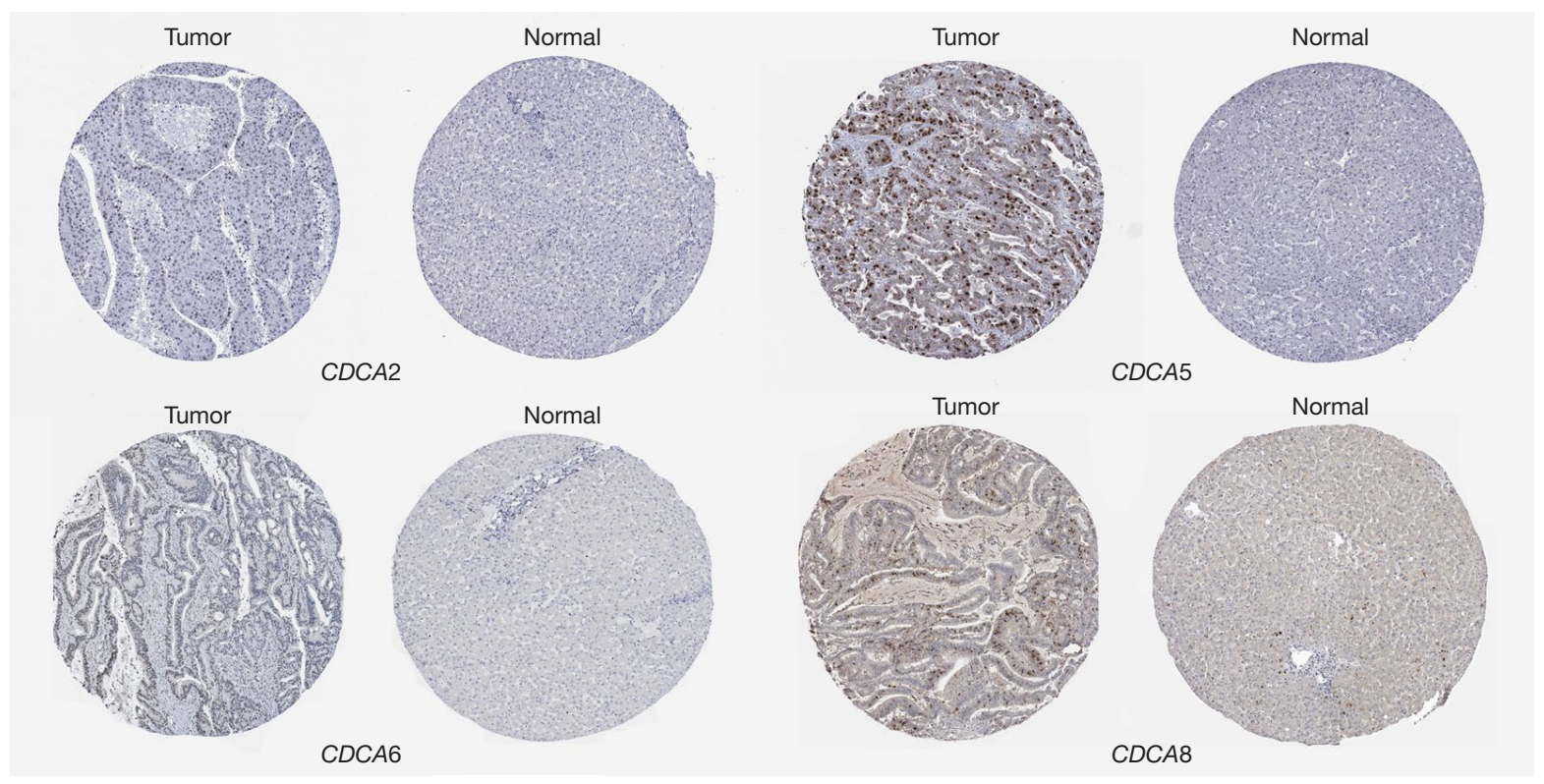

Figure 3 The protein levels of $C D C A$ family members in HCC tissues and normal tissues with IHC staining. $C D C A$, cell division cycleassociated. Original magnification: $\times 100$.



$\mathrm{P}<0.0001$

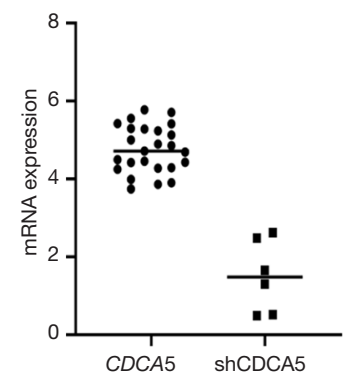

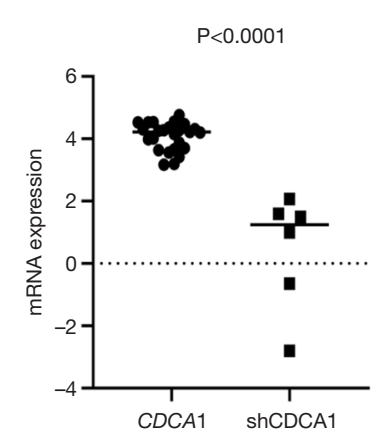

$P<0.0001$

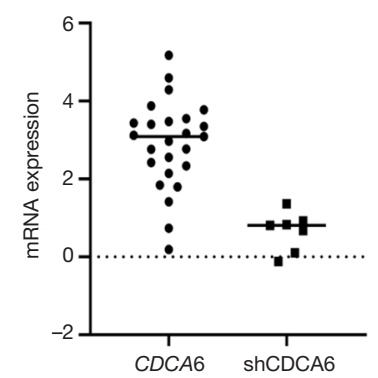

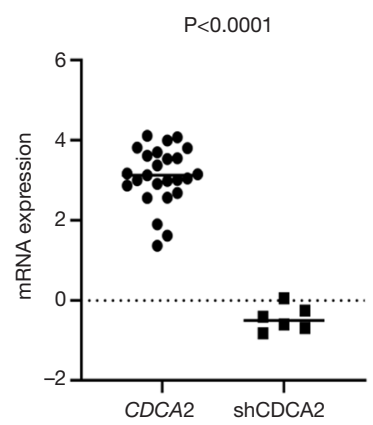

$P<0.0001$

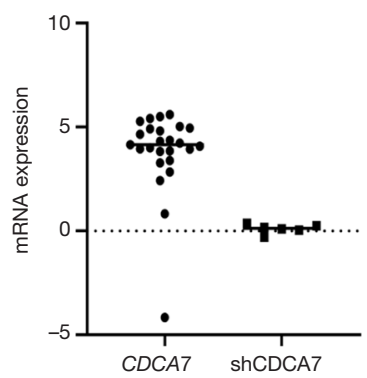

$\mathrm{P}<0.0001$

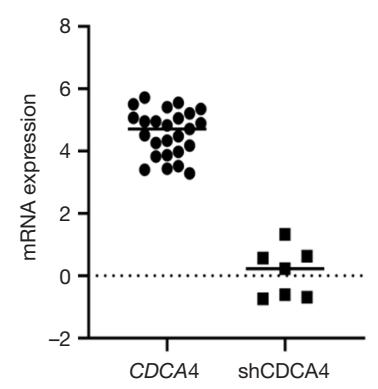

$P<0.0001$



Figure 4 The expression levels of all $C D C A$ family members in HCC cell lines and the comparison of expression levels of $C D C A$ family members in HCC cell lines before and after knockdown. CDCA, cell division cycle-associated. 

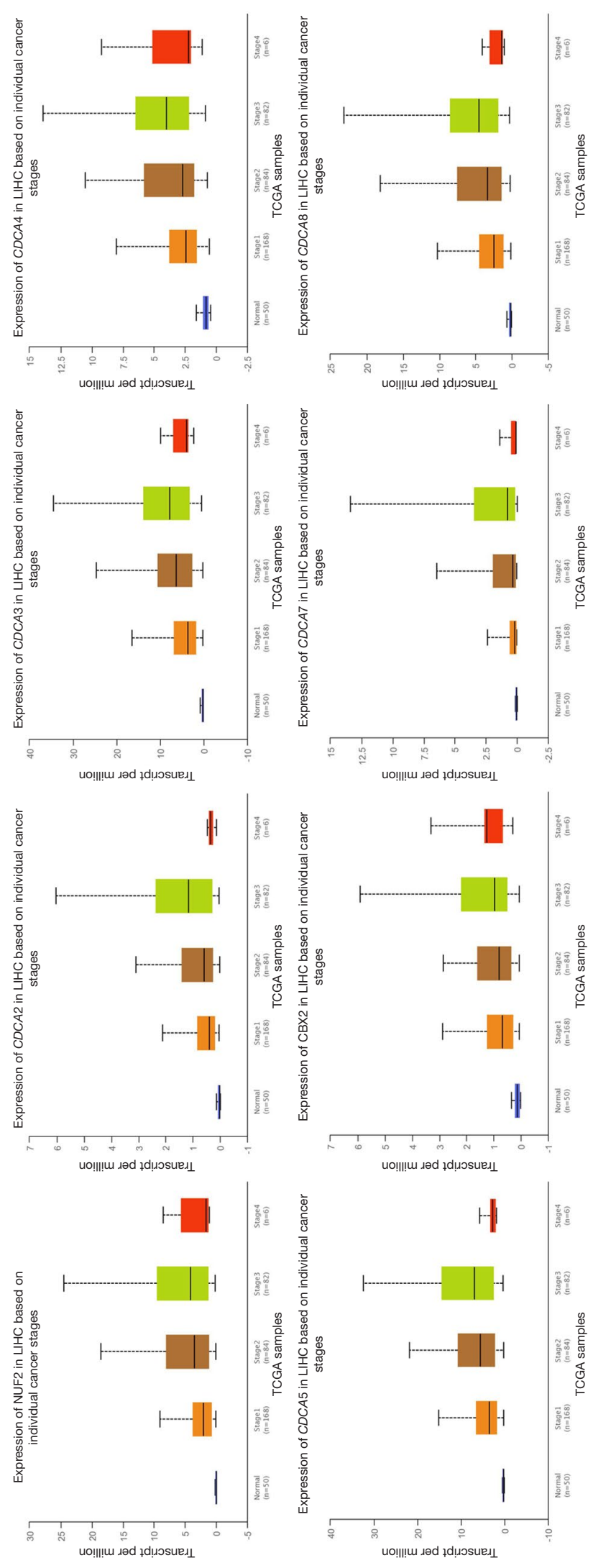

proteins; GO:0034508: centromere complex assembly; GO:0051301: cell division; R-HSA-3214858: RMTs methylate histone arginines; and R-HSA-68875: Mitotic Prophase.

\section{Discussion}

According to reports, $C D C A$ gene abnormalities occur in many cancers $(4,5)$. Despite the carcinogenetic and prognostic functions of $C D C A$ family members in several cancers having been well documented (31-33), an in-depth bioinformatics analysis of their roles in HCC had yet to be performed. Therefore, this study analyzed the expressions and mutations as well as the prognostic values of the $C D C A$ family genes in HCC.

$C D C A 1$ is a crucial constituent of the NDC 80 kinetochore complex, which is necessary for kinetochoremicrotubule connection as well as chromosome separation (34). Previous research showed that CDCA1 promoted the growth and inhibited the apoptosis of HCC cells (35). Wang et al. further showed that high expression of $C D C A 1$ is significantly related to the poor survival of patients with HCC, and $C D C A 1$ therefore holds promise as a prognostic biomarker to aid in the accurate prediction of early recurrence of HCC after surgical treatment (34). In the present study, the expression of CDCA1 mRNA in HCC tissues was significantly higher than that in normal tissues and was significantly correlated with the individual cancer stage; this finding was consistent with the results of previous studies. Moreover, high CDCA1 mRNA expression was also significantly related to poor survival in HCC patients, indicating that $C D C A 1$ participates in HCC tumorigenesis.

$\mathrm{Up}$ to now, researchers have gained little insight into the performance and function of $C D C A 2$ in HCC. It has been reported that $C D C A 2$ is a cell cycle-related protein, the expression of which is related to other members of the $C D C A$ gene family (36). According to previous studies, $C D C A 2$ plays a key role in regulating the expression of PP $1 \gamma$-dependent essential DNA damage responses in the cell cycle as well as preserving the characteristic chromosome structure for transiting to interphase $(7,37)$. Several studies have revealed $C D C A 2$ to be highly expressed in tissue samples from patients with oral squamous cell carcinoma, neuroblastoma, and adenocarcinoma of the lung (38-40). Recent research indicated that CDCA2 might target $C C N D 1$, at least in part by activating the PI3K/AKT pathway to promote colorectal carcinoma cell proliferation and tumorigenesis (41). Our current study showed that 

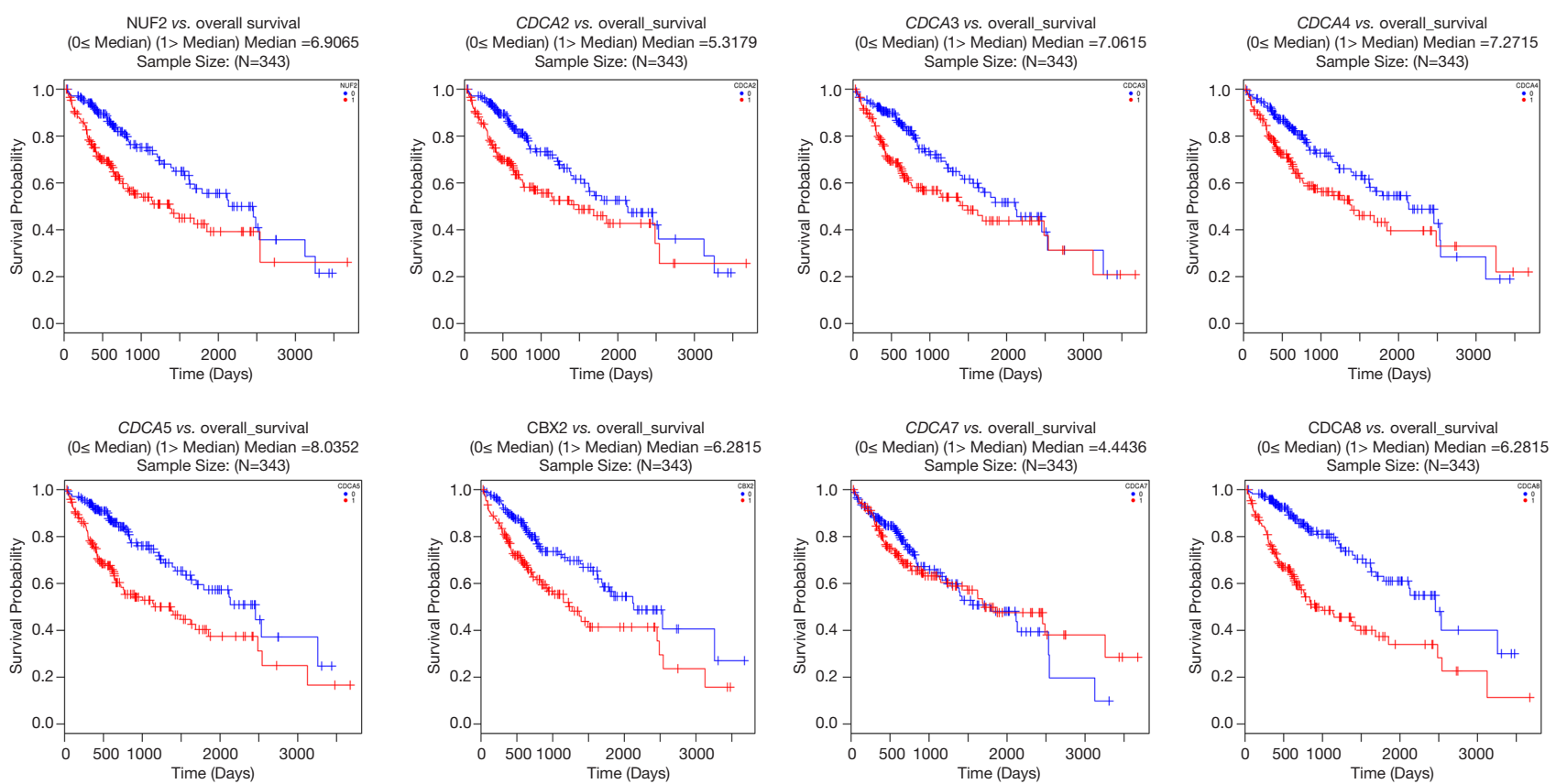

Figure 6 The prognostic value of the $C D C A$ gene expression in HCC patients. $C D C A$, cell division cycle-associated; HCC, hepatocellular carcinoma.

CDCA2 was expressed more highly in HCC tissues than in normal tissues. Furthermore, we found that $C D C A 2$ expression was related to HCC stage. Among all the HCC patients, a high expression of $C D C A 2$ was significantly related to poor OS, which indicated that $C D C A 2$ has carcinogenic effects in HCC.

$C D C A 3$ plays a significant role in cell mitosis and control of the $\mathrm{G} 1$ phase (8). The involvement of $C D C A 3$ has been reported in lung cancer, prostate cancer, and oral squamous cell carcinoma (42-44). Furthermore, in colorectal cancer, $C D C A 3$ is upregulated, and its upregulation is correlated with the proliferation and apoptosis of cancer cells. This effect may be achieved in colorectal cancer through activation of the nuclear factor-kappa B (NF- $\mathrm{KB})$ signaling pathway by $C D C A 3$ via interaction with TRAF2 (8). Studies have also shown that $C D C A 3$ expression is elevated in liver cancer and may be involved in cell proliferation, migration, invasion, and apoptosis $(15,16)$. In our present study, $C D C A 3$ expression was significantly higher in HCC tissues than in adjacent normal tissues, and the mRNA expression of $C D C A 3$ was strongly related to cancer stage. Furthermore, a high expression level of $C D C A 3$ was found to be significantly correlated with poor OS in patients with HCC.

$C D C A 4$ was first discovered when mouse hematopoietic stem cells were screened against a reduced cDNA library. It was named hematopoietic progenitor protein (HEPP) on the basis of its preferred expression in adult bone marrow hematopoietic progenitor cells (45). Alderman et al. found $C D C A 4$ to be highly expressed in melanoma and to be significantly associated with poor prognosis. Their study also found that microRNA-15a can directly regulate the expression of the CDCA4 gene, thereby regulating the proliferation of melanoma cells (46). In this study, similar tumorigenicity of CDCA4 was demonstrated in HCC. According to the results of our study, the mRNA expression of $C D C A 4$ in HCC tissues was higher than that in adjacent normal tissues, and its expression level was significantly related to cancer stage and OS in HCC patients.

CDCA5 is also considered to be an oncogene due to its overexpression in multiple cancers $(11,18)$. A previous study showed that the CDCA5 gene is extremely important for the genesis and progression of HCC, in which it is highly expressed and is significantly associated with tumor progression and poor prognosis (18). Similar to observations made in a previous study, the mRNA expression of $C D C A 5$ was found to be higher in HCC tissues in our study and was significantly correlated with cancer stage. A higher expression of $C D C A 5$ was also remarkably related to shorter OS in HCC patients. 


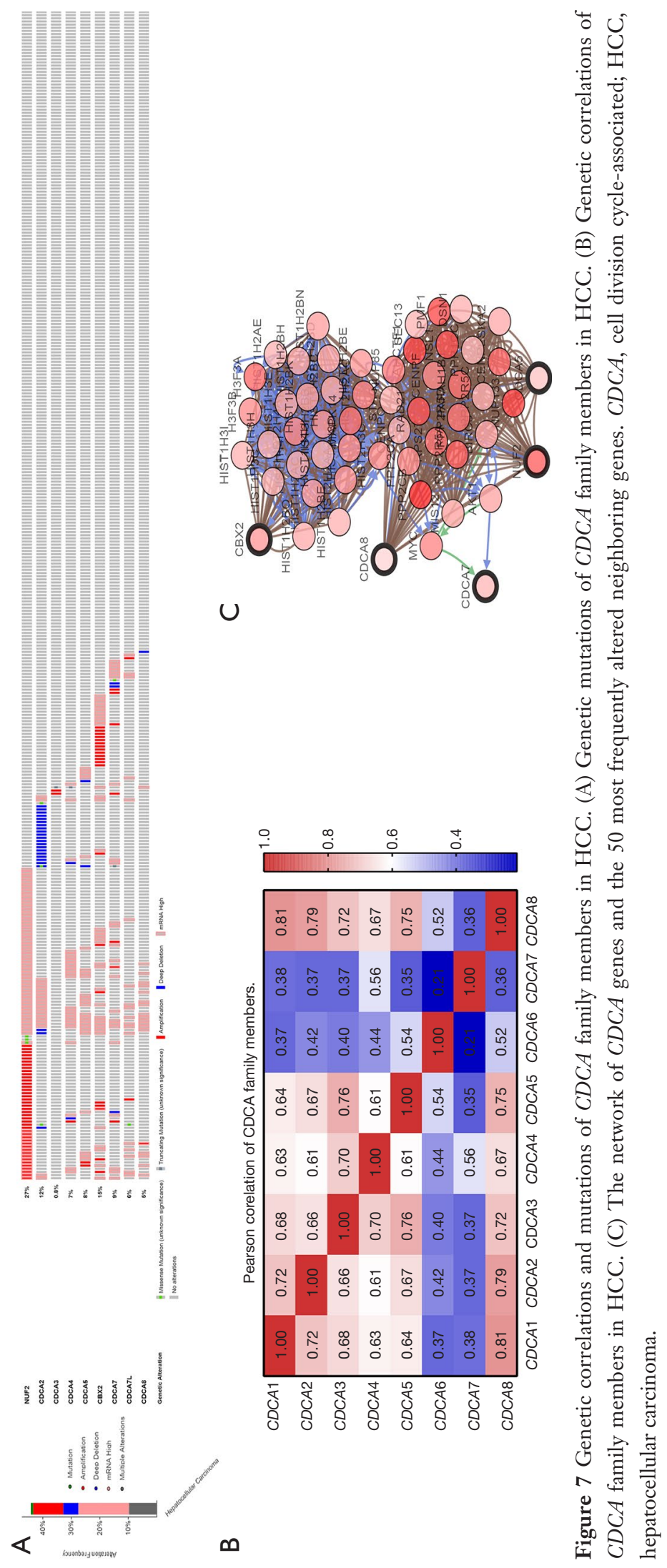




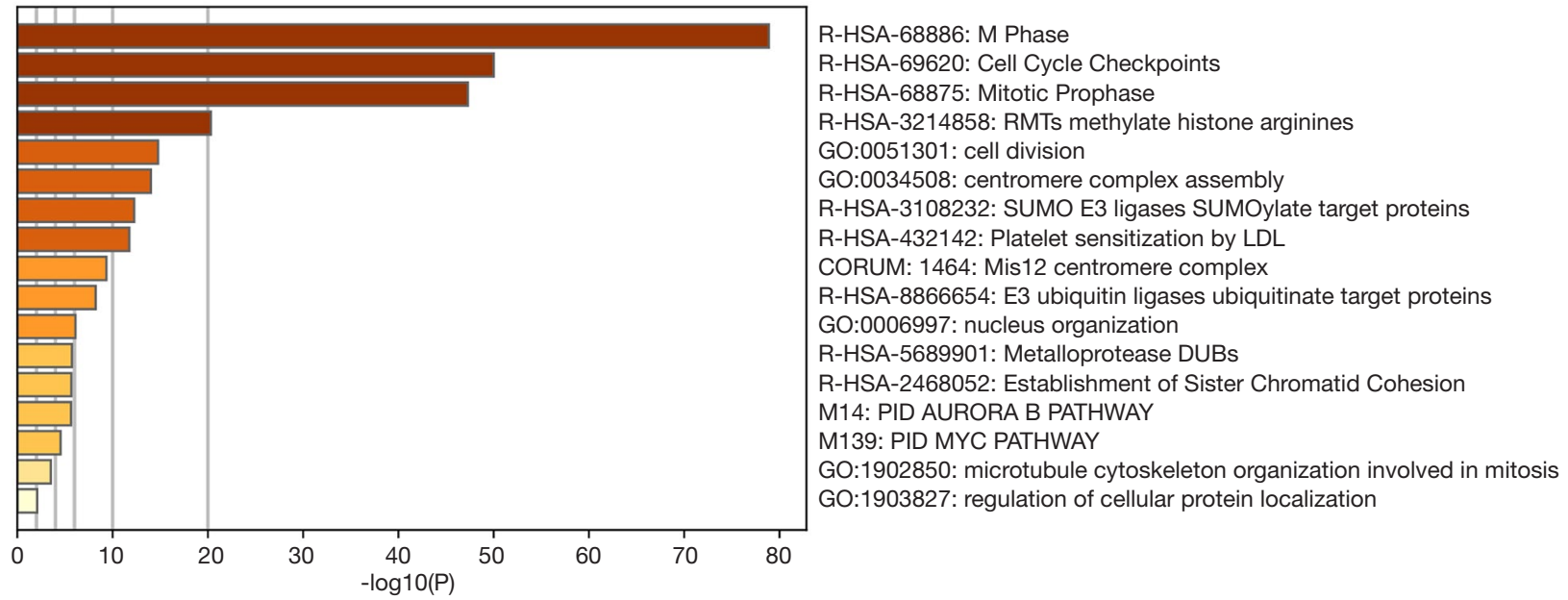

Figure 8 The functions of $C D C A$ genes and genes significantly related to $C D C A$ mutations. $C D C A$, cell division cycle-associated.

As a vital component of polycomb repressive complexes 1 (PRC1), CDCA6 is involved in the gene expression and heterochromatin regulation (47). A previous study showed that, in breast cancer, CDCA6 expression is positively correlated to tumor size and TNM stage (48). CDCA6 has been reported as a potential drug target because its expression in association with adverse clinical outcomes in prostate cancer patients (49). In this study, our results were consistent with these previous founding. We found that $C D C A 6$ was overexpressed in HCC tissues and high expression of it correlated with poor outcome in HCC patients, indicating that $C D C A 6$ is an oncogene.

$C D C A 7$ has been regarded as a cMyc target gene (13). A recent study found that the abnormal upregulation of $C D C A 7$ in patients with breast cancer was related to a dismal prognosis and induced the progression of Enhancer of Zeste Homolog 2 (EZH2)-mediated triple-negative breast cancer (32). The results of our current study showed that $C D C A 7$ was expressed at a higher level in HCC tissues compared with adjacent normal tissues, and the expression level was significantly related to cancer stage. It was also found that in HCC patients, high CDCA7 mRNA expression was related to poor OS.

As an important regulatory gene during mitosis, CDCA8 has been found to have enhanced transcriptional activity in embryos, embryonic stem cells, and cancer cells. Meanwhile, CDCA8 knockdown can effectively inhibit the proliferation of lung cancer cells, colon cancer cells, and human embryonic stem cells, and can promote and induce cell differentiation (50-52). A study by Jiao et al. revealed that the overexpression of $C D C A 8$ in breast cancer reduced patient survival (53). In the present study, $C D C A$ was expressed at significantly higher levels in HCC tissues, and its expression was correlated with disease stage. Accordingly, higher expression levels of $C D C A 8$ were also associated with shorter OS in HCC patients.

In this study, GO and KEGG analyses were also carried out to identify the associations of $C D C A$ genes and the most frequently altered linked genes with HCC initiation and prognosis. According to our research, closer attention should be paid to the following pathways: R-HSA-68886: $\mathrm{M}$ phase; R-HSA-69620: cell cycle checkpoints; R-HSA-68875: mitotic prophase; R-HSA-3214858: RMTs methylate histone arginines; GO:0051301: cell division; GO:0034508: centromere complex assembly; R-HSA-3108232: SUMO E3 ligases SUMOylate target proteins; and R-HSA-432142: Platelet sensitization by LDL.

Some limitations of the present study should be noted. First, we analyzed data retrieved from online databases, and further studies with larger sample sizes are needed to validate our findings. Also, we failed to investigate the underlying clinical roles and mechanisms of distinct $C D C A$ genes in HCC, which demands further research.

\section{Conclusions}

In conclusion, we systematically investigated the prognostic value and expression levels of $C D C A$ genes in HCC, which clarified the heterogeneity as well as complexity of the biological properties of HCC at the molecular level. According to our observations, overexpression of $C D C A$ genes in HCC tissues likely plays a considerable role in 
HCC oncogenesis. Moreover, overexpression of $C D C A$ genes may also serve as a molecular marker to improve prognostic accuracy and survival for patients with HCC.

\section{Acknowledgments}

Funding: This work was funded by grants from the Science and Technology Program of Guangzhou, China (No. 201803010071) and the Rural Science and Technology Commissioner Program of Guangdong Province, China (No. 202010130627).

\section{Footnote}

Reporting Checklist: The authors have completed the MDAR reporting checklist. Available at http://dx.doi.org/10.21037/ jgo-21-110

Conflicts of Interest: All authors have completed the ICMJE uniform disclosure form (available at http://dx.doi. org/10.21037/jgo-21-110). The authors have no conflicts of interest to declare.

Ethical Statement: The authors are accountable for all aspects of the work in ensuring that questions related to the accuracy or integrity of any part of the work are appropriately investigated and resolved. This research was approved by the institutional ethics committee of Sun Yat-Sen University Cancer Center and was carried out in accordance with the principles of the Helsinki Declaration (as revised in 2013). All data used in this study were retrieved from publicly available sources, so there was no requirement to obtain informed participant consent.

Open Access Statement: This is an Open Access article distributed in accordance with the Creative Commons Attribution-NonCommercial-NoDerivs 4.0 International License (CC BY-NC-ND 4.0), which permits the noncommercial replication and distribution of the article with the strict proviso that no changes or edits are made and the original work is properly cited (including links to both the formal publication through the relevant DOI and the license). See: https://creativecommons.org/licenses/by-nc-nd/4.0/.

\section{References}

1. Bray F, Ferlay J, Soerjomataram I, et al. Global cancer statistics 2018: GLOBOCAN estimates of incidence and mortality worldwide for 36 cancers in 185 countries. CA Cancer J Clin 2018;68:394-424.

2. Chen $\mathrm{W}$, Zheng R, Baade PD, et al. Cancer statistics in China, 2015. CA Cancer J Clin 2016;66:115-32.

3. Bruix J, Sherman M, American Association for the Study of Liver D. Management of hepatocellular carcinoma: an update. Hepatology 2011;53:1020-2.

4. Spruck CH, Strohmaier HM. Seek and destroy: SCF ubiquitin ligases in mammalian cell cycle control. Cell Cycle 2002;1:250-4.

5. Phan NN, Wang CY, Li KL, et al. Distinct expression of CDCA3, CDCA5, and CDCA8 leads to shorter relapse free survival in breast cancer patient. Oncotarget 2018;9:6977-92.

6. Tokuzumi A, Fukushima S, Miyashita A, et al. Cell division cycle-associated protein 1 as a new melanoma-associated antigen. J Dermatol 2016;43:1399-405.

7. Vagnarelli P. Repo-man at the intersection of chromatin remodelling, DNA repair, nuclear envelope organization, and cancer progression. Adv Exp Med Biol 2014;773:401-14.

8. Zhang $\mathrm{W}, \mathrm{Lu} \mathrm{Y}, \mathrm{Li}$ X, et al. CDCA3 promotes cell proliferation by activating the NF-kappaB/cyclin D1 signaling pathway in colorectal cancer. Biochem Biophys Res Commun 2018;500:196-203.

9. Hayashi R, Goto Y, Ikeda R, et al. CDCA4 is an E2F transcription factor family-induced nuclear factor that regulates E2F-dependent transcriptional activation and cell proliferation. J Biol Chem 2006;281:35633-48.

10. Tategu $M$, Nakagawa $H$, Hayashi R, et al. Transcriptional co-factor CDCA4 participates in the regulation of JUN oncogene expression. Biochimie 2008;90:1515-22.

11. Chang IW, Lin VC, He HL, et al. CDCA5 overexpression is an indicator of poor prognosis in patients with urothelial carcinomas of the upper urinary tract and urinary bladder. Am J Transl Res 2015;7:710-22.

12. Clermont PL, Sun L, Crea F, et al. Genotranscriptomic meta-analysis of the Polycomb gene CBX2 in human cancers: initial evidence of an oncogenic role. Br J Cancer 2014;111:1663-72.

13. Jimenez PR, Martin-Cortazar C, Kourani O, et al. CDCA7 is a critical mediator of lymphomagenesis that selectively regulates anchorage-independent growth. Haematologica 2018;103:1669-78.

14. Higuchi T, Uhlmann F. Cell cycle: passenger acrobatics. Nature 2003;426:780-1.

15. Hu Q, Fu J, Luo B, et al. OY-TES-1 may regulate the malignant behavior of liver cancer via NANOG, CD9, 
CCND2 and CDCA3: a bioinformatic analysis combine with RNAi and oligonucleotide microarray. Oncol Rep 2015;33:1965-75.

16. Jang SI, Lee YW, Cho CK, et al. Identification of Target Genes Involved in the Antiproliferative Effect of EnzymeModified Ginseng Extract in HepG2 Hepatocarcinoma Cell. Evid Based Complement Alternat Med 2013;2013:502568.

17. Li B, Pu K, Wu X. Identifying novel biomarkers in hepatocellular carcinoma by weighted gene coexpression network analysis. J Cell Biochem 2019. [Epub ahead of print].

18. Tian Y, Wu J, Chagas C, et al. CDCA5 overexpression is an Indicator of poor prognosis in patients with hepatocellular carcinoma (HCC). BMC Cancer 2018;18:1187.

19. Chen H, Chen J, Zhao L, et al. CDCA5, Transcribed by E2F1, Promotes Oncogenesis by Enhancing Cell Proliferation and Inhibiting Apoptosis via the AKT Pathway in Hepatocellular Carcinoma. J Cancer 2019;10:1846-54.

20. Rhodes DR, Yu J, Shanker K, et al. ONCOMINE: a cancer microarray database and integrated data-mining platform. Neoplasia 2004;6:1-6.

21. Chandrashekar DS, Bashel B, Balasubramanya SAH, et al. UALCAN: A Portal for Facilitating Tumor Subgroup Gene Expression and Survival Analyses. Neoplasia 2017;19:649-58.

22. Thul PJ, Lindskog C. The human protein atlas: A spatial map of the human proteome. Protein Sci 2018;27:233-44.

23. Barretina J, Caponigro G, Stransky N, et al. The Cancer Cell Line Encyclopedia enables predictive modelling of anticancer drug sensitivity. Nature 2012;483:603-7.

24. Vasaikar SV, Straub P, Wang J, et al. LinkedOmics: analyzing multi-omics data within and across 32 cancer types. Nucleic Acids Res 2018;46:D956-63.

25. Cancer Genome Atlas N. Comprehensive molecular portraits of human breast tumours. Nature 2012;490:61-70.

26. Cerami E, Gao J, Dogrusoz U, et al. The cBio cancer genomics portal: an open platform for exploring multidimensional cancer genomics data. Cancer Discov 2012;2:401-4.

27. Zhou Y, Zhou B, Pache L, et al. Metascape provides a biologist-oriented resource for the analysis of systemslevel datasets. Nat Commun 2019;10:1523.

28. Chen X, Cheung ST, So S, et al. Gene expression patterns in human liver cancers. Mol Biol Cell 2002;13:1929-39.
29. Wurmbach E, Chen YB, Khitrov G, et al. Genomewide molecular profiles of $\mathrm{HCV}$-induced dysplasia and hepatocellular carcinoma. Hepatology 2007;45:938-47.

30. Roessler S, Jia HL, Budhu A, et al. A unique metastasis gene signature enables prediction of tumor relapse in early-stage hepatocellular carcinoma patients. Cancer Res 2010;70:10202-12.

31. Zhao Z, Li C, Song B, et al. pH low insertion peptide mediated cell division cycle-associated protein 1 -siRNA transportation for prostatic cancer therapy targeted to the tumor microenvironment. Biochem Biophys Res Commun 2018;503:1761-7.

32. Ye L, Li F, Song Y, et al. Overexpression of CDCA7 predicts poor prognosis and induces EZH2-mediated progression of triple-negative breast cancer. Int J Cancer 2018;143:2602-13.

33. Kobayashi Y, Takano A, Miyagi Y, et al. Cell division cycle-associated protein 1 overexpression is essential for the malignant potential of colorectal cancers. Int J Oncol 2014;44:69-77.

34. Wang Y, Tan PY, Handoko YA, et al. NUF2 is a valuable prognostic biomarker to predict early recurrence of hepatocellular carcinoma after surgical resection. Int J Cancer 2019; 145:662-70.

35. Liu Q, Dai SJ, Li H, et al. Silencing of NUF2 inhibits tumor growth and induces apoptosis in human hepatocellular carcinomas. Asian Pac J Cancer Prev 2014;15:8623-9.

36. Walker MG. Drug target discovery by gene expression analysis: cell cycle genes. Curr Cancer Drug Targets 2001;1:73-83.

37. Peng A, Lewellyn AL, Schiemann WP, et al. Repo-man controls a protein phosphatase 1-dependent threshold for DNA damage checkpoint activation. Curr Biol 2010;20:387-96.

38. Krasnoselsky AL, Whiteford CC, Wei JS, et al. Altered expression of cell cycle genes distinguishes aggressive neuroblastoma. Oncogene 2005;24:1533-41.

39. Uchida F, Uzawa K, Kasamatsu A, et al. Overexpression of CDCA2 in human squamous cell carcinoma: correlation with prevention of G1 phase arrest and apoptosis. PLoS One 2013;8:e56381.

40. Shi R, Zhang C, Wu Y, et al. CDCA2 promotes lung adenocarcinoma cell proliferation and predicts poor survival in lung adenocarcinoma patients. Oncotarget 2017;8:19768-79.

41. Feng Y, Qian W, Zhang Y, et al. CDCA2 promotes the proliferation of colorectal cancer cells by activating the 
$\mathrm{AKT} / \mathrm{CCND} 1$ pathway in vitro and in vivo. BMC Cancer 2019; 19:576.

42. Chen J, Zhu S, Jiang N, et al. HoxB3 promotes prostate cancer cell progression by transactivating CDCA3. Cancer Lett 2013;330:217-24.

43. Adams MN, Burgess JT, He Y, et al. Expression of CDCA3 Is a Prognostic Biomarker and Potential Therapeutic Target in Non-Small Cell Lung Cancer. J Thorac Oncol 2017;12:1071-84.

44. Uchida F, Uzawa K, Kasamatsu A, et al. Overexpression of cell cycle regulator CDCA3 promotes oral cancer progression by enhancing cell proliferation with prevention of G1 phase arrest. BMC Cancer 2012;12:321.

45. Abdullah JM, Jing X, Spassov DS, et al. Cloning and characterization of Hepp, a novel gene expressed preferentially in hematopoietic progenitors and mature blood cells. Blood Cells Mol Dis 2001;27:667-76.

46. Alderman C, Sehlaoui A, Xiao Z, et al. MicroRNA$15 \mathrm{a}$ inhibits the growth and invasiveness of malignant melanoma and directly targets on CDCA4 gene. Tumour Biol 2016;37:13941-50.

47. Mao J, Tian Y, Wang C, et al. CBX2 Regulates Proliferation and Apoptosis via the Phosphorylation of YAP in Hepatocellular Carcinoma. J Cancer 2019;10:2706-19.

Cite this article as: Tao Q, Chen S, Liu J, Zhao P, Jiang L, Tu X, Tang X, Liu Z, Yasheng A, Tuerxun K, Zheng Y. The roles of the cell division cycle-associated gene family in hepatocellular carcinoma. J Gastrointest Oncol 2021;12(2):781794. doi: 10.21037/jgo-21-110
48. Chen WY, Zhang XY, Liu T, et al. Chromobox homolog 2 protein: A novel biomarker for predicting prognosis and Taxol sensitivity in patients with breast cancer. Oncol Lett 2017;13:1149-56.

49. Clermont PL, Crea F, Chiang YT, et al. Identification of the epigenetic reader CBX2 as a potential drug target in advanced prostate cancer. Clin Epigenetics 2016;8:16.

50. Hayama S, Daigo Y, Yamabuki T, et al. Phosphorylation and activation of cell division cycle associated 8 by aurora kinase B plays a significant role in human lung carcinogenesis. Cancer Res 2007;67:4113-22.

51. Wang Y, Zhao Z, Bao X, et al. Borealin/Dasra B is overexpressed in colorectal cancers and contributes to proliferation of cancer cells. Med Oncol 2014;31:248.

52. Dai C, Miao CX, Xu XM, et al. Transcriptional activation of human CDCA8 gene regulated by transcription factor NF-Y in embryonic stem cells and cancer cells. J Biol Chem 2015;290:22423-34.

53. Jiao DC, Lu ZD, Qiao JH, et al. Expression of CDCA8 correlates closely with FOXM1 in breast cancer: public microarray data analysis and immunohistochemical study. Neoplasma 2015;62:464-9.

(English Language Editor: J. Reynolds) 